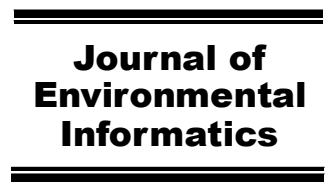

www.iseis.org/jei

\title{
PLL Performance Evaluation Considering Power System Dynamics for Grid Connection of Renewable Power Generation
}

\author{
W. J. Du* X. Chen, and H. F. Wang \\ The State Key Laboratory of Alternate Electric Power Systems with New Energy Resources, North China Electric Power University, Changping \\ District, Beijing, China, 102206
}

Received 10 October 2015; revised 3 January 2018; accepted 28 March 2018; published online 26 September 2018

\begin{abstract}
Successful vector control for grid connection of renewable power generation relies on accurate and fast tracking of the voltage phase at the point of common coupling using a phase-locked loop (PLL). In this study, we propose a method to evaluate the effect of power system dynamics on the PLL performance. Simulation results demonstrate that the PLL performance is considerably affected by the power system dynamics, which is not observable under conventional test conditions considering a PLL connected to a three-phase voltage source. Therefore, we proposed an improved evaluation method considering the same source but integrating the system dynamics during evaluation. We expect that the proposed method will assist in the design of PLLs to improve the phase tracking of the voltage at the point of common coupling.
\end{abstract}

Keywords: first-swing stability, low-frequency oscillation, phase-locked loop, power system dynamics, power system simulation

\section{Introduction}

Driven by environmental concerns, the development of renewable power generation has rapidly increased over the last two decades. Moreover, renewable energy is expected to become the major generation source in future power systems. Currently, grid connection of large-scale renewable power generation relies on vector control of voltage source converters (VSCs). To implement vector control, the voltage phase at the point of common coupling (PCC) must be tracked using phase-locked loop (PLL) (Pena et al., 1996; Peng and Lai, 1997; Cole et al., 2010; Li et al., 2012;). Hence, the PLL is an essential component for grid connection of large-scale renewable energy and supports environmental benefits including clean power generation and reduction in the use of fossil fuels (Huang and Chang, 2003).

Several PLL design schemes have been proposed. Among them, the most widely used scheme is the synchronous reference frame PLL (SRF-PLL) (Kaura and Blasko, 1997; Chung, 2000; Timbus et al., 2005; Shi and Crow, 2008; Kesler and Ozdemir, 2011), which provides a simple structure and fast response under normal operation of three-phase power systems. However, the performance of the SRF-PLL is com-

${ }^{*}$ Corresponding author. Tel.: +086 15510040887; fax: +086 1061771611.
E-mail address: ddwenjuan@gmail.com (W. J. Du).

ISSN: 1726-2135 print/1684-8799 online

(C) 2018 ISEIS All rights reserved. doi:10.3808/jei.201800396 promised when operating under unbalanced conditions, and it can even fail to track the phase of a positive-sequence voltage under severe unbalance.

To overcome this problem, the decoupled double SRFPLL (DDSRF-PLL) has been proposed for operation under unbalanced conditions (Rodríguez et al., 2005; Rodríguez et al., 2007; Hadjidemetriou et al., 2013). In fact, the DDSRFPLL tracks the phase of both positive- and negative-sequence voltages and can be used to improve VSC vector control (Song and Nam, 1999; Xu et al., 2005; Sinsukthavorn et al., 2012). However, like the SRF-PLL, the DDSRF-PLL relies on a proportional-integral (PI) feedback control, which may limit the tracking speed. In contrast, a PLL design scheme based on the fast Fourier transform (FFT-PLL) has been recently proposed (Da Silva et al., 2010; Lee et al., 2011, 2014). The FFTPLL tracks the voltage phase in a three-phase system by applying the FFT instead of PI feedback control. However, the tracked phase-A voltage is only equal to that of the positivesequence three-phase voltage when the system is balanced.

Overall, the development of PLLs should be thoroughly evaluated in terms of performance to improve and validate the proposed designs. This evaluation is usually performed through either simulation or laboratory experiments by connecting the PLL to a three-phase voltage source. Then, the PLL performance for voltage phase tracking at the PCC is determined from the response to step changes in the voltage. In some cases, this PLL tracking performance evaluation retrieves similar results to those in a real PLL connected to a power 
system. For instance, the PCC can be assumed as an infinite bus in distri- buted generation, whose voltage can be modeled as an ideal three-phase voltage source. However, when the power system dynamics considerably alters the PCC voltage phase, these effects should be also considered. In fact, lowfrequency oscillation may occur during transients in power systems (Du et al., 2015, 2016). In addition, large disturbances can drastically change the power angle among synchronous generators (SGs) (Wu et al., 2014). This type of power system dynamics might affect the PLL tracking performance as unexpected variations in the voltage phase can occur. Therefore, the PLL performance evaluation should consider the operation in a power system, rather than in a three-phase voltage source, to suitably assess the PLL capabilities under dynamic variations and different faults. To the best of our knowledge, this is the first work that considers the power system dynamics to evaluate the PLL performance.

The main contributions of this study can be summarized as follows. We compare the PLL performance when connected to a three-phase voltage source and to a power system considering its dynamics. The abovementioned PLL design schemes are evaluated to determine their tracking performance. The evaluation results highlight the importance of considering the power system dynamics on the PLL performance. As a result, we propose a new method for PLL performance evaluation that integrates the power system dynamics to the method based on the three-phase voltage source. Therefore, the proposed evaluation method is fast, efficient, and simple to implement on any PLL structure.

This paper is organized as follows. In Section 2, the conventional test results obtained by connecting the PLL to a theoretical infinite bus are presented. Section 3 presents the simulation results when the power system dynamics are considered. The comparison of the results from Sections 2 and 3 indicates that the evaluation method considering the infinite bus is not suitable for an accurate PLL assessment, and hence we propose and verify an improved evaluation method incurporating the power system dynamics in Section 4. Finally, we draw conclusions in Section 5.

\section{Performance Evaluation of PLL Connected to Ideal Three-phase Voltage Source}

In this section, we briefly discuss three representative PLL designs, namely, the SRF-, DDSRF-, and FFT-PLL and evaluate their performance using the method based on an ideal three-phase voltage source to determine the limitations of this approach.

\subsection{Evaluated PLL Designs}

The structure of the SRF-PLL is shown in Figure 1 (Shi and Crow, 2008). This PLL is based on the $a b c-d q$ transformation to obtain $V_{q 1}$. Under unbalanced condition, $V_{q 1}$ may be polluted by the negative-sequence component of voltage source $V_{a b c}$, which varies at double of the nominal frequency. Usually, a lowpass filter is used to eliminate this high-frequency component and obtain $V_{q}$.

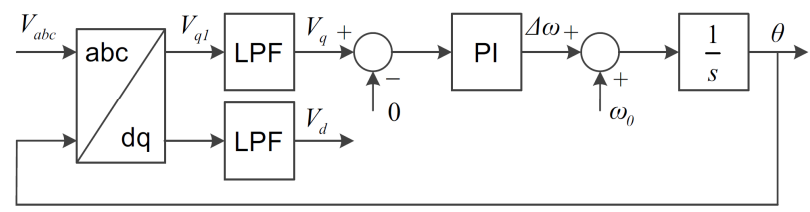

Figure 1. Structure of SRF-PLL (LPF, lowpass filter).

Next, the structure of the DDSRF-PLL is shown in Figure 2 (Rodríguez et al., 2007). This PLL is characterized by double reference frames, which enable calculation of the $d$ - $q$-axis components of both the positive- and negativesequence voltages. By using a specialized algorithm (Rodríguez et al., 2007), the accurate value of $V_{q^{+}}$for the positivesequence voltage can be obtained.

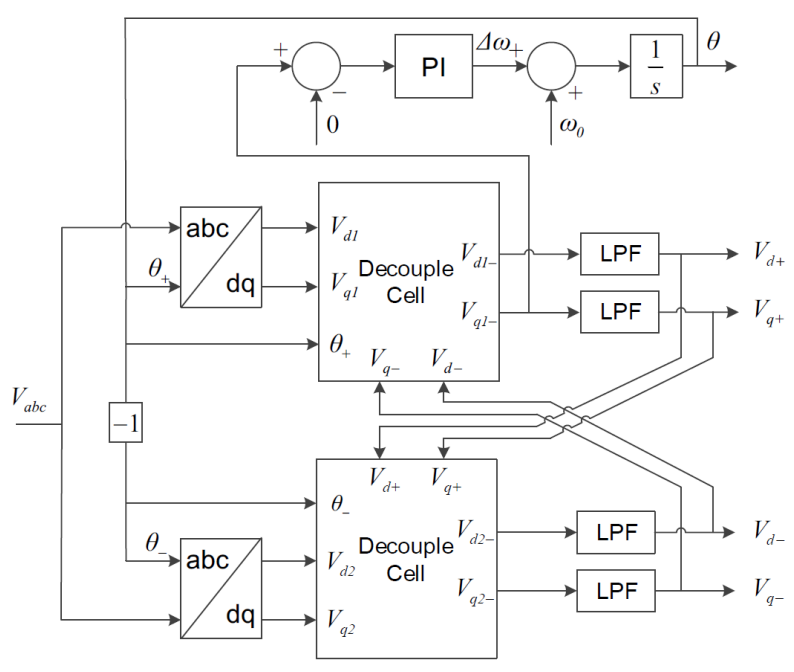

Figure 2. Structure of DDSRF-PLL.

Finally, the structure of the FFT-PLL is shown in Figure 3 (Lee et al., 2014). Unlike the SRF- and DDSRF-PPL, the FFT-PLL does not include a PI feedback controller. In addition, the FFT-PLL aims only to lock the phase of phase A, rather than that of the three-phase voltage.

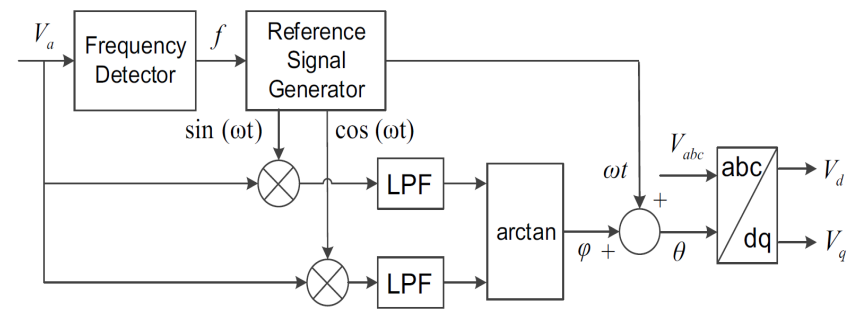

Figure 3. Structure of FFT-PLL. 


\subsection{Performance of PLL Connected to Ideal Three-phase Voltage Source}

Vector control satisfying $V_{q}=0$ allows to realize decoupled control of the active and reactive power. Therefore, any deviation of $V_{q}$ from zero indicates the inability of the PLL to track the PCC voltage phase. The usual evaluation of PLL performance considers its connection to a three-phase voltage source. Then, the $V_{q}$ deviation caused by the PLL can be assessed through simulations or laboratory experiments.

We evaluated the performance of the three representative PLL designs presented in Section 2.1 through simulation by applying the following three-phase voltage:

$$
\left\{\begin{array}{l}
V_{a}=V_{0} \cos \left(\omega_{0} t+\Delta \delta\right) \\
V_{b}=V_{0} \cos \left(\omega_{0} t-2 \pi / 3+\Delta \delta\right) \\
V_{c}=V_{0} \cos \left(\omega_{0} t+2 \pi / 3+\Delta \delta\right)
\end{array}\right.
$$

Then, we applied the following step decrease in the phase of the three-phase voltage at $0.4 \mathrm{~s}$ :

$\Delta \delta=\left\{\begin{array}{l}0 \quad t<0.4 \text { second } \\ -\frac{\pi}{3} \quad t>0.4 \text { second }\end{array}\right.$

Figure 4 shows the evaluation results, where both the SRF- and DDSRF-PLL produce similar deviations in $V_{q}$. In fact, in approximately $0.15 \mathrm{~s}$, both provide accurate tracking of the voltage phase, i.e., $V_{q}=0$. Still, the performance of the FFT-PLL is better because it tracks the voltage phase in approximately $0.1 \mathrm{~s}$.

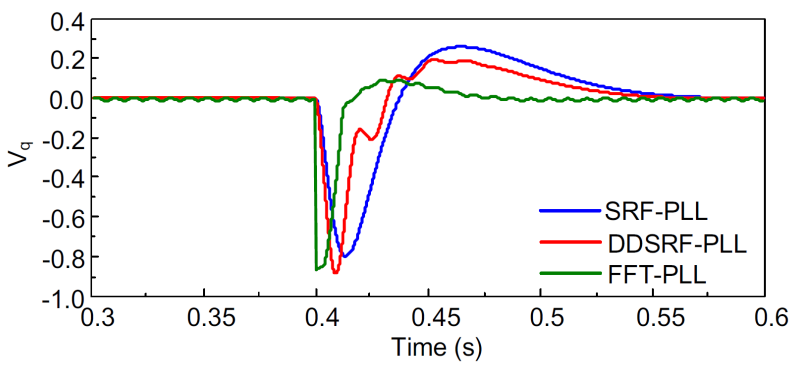

Figure 4. Performance of different PLLs after a step phase decrease of the three-phase voltage.

The performance of the SRF- and DDSRF-PLL is highly affected by the parameters of the PI controller. In the abovementioned tests, where the PLL operates in a balanced three-phase condition, the PI parameters were tuned to retrieve a similar performance among the PLLs and kept constant throughout the simulations in this study.

To evaluate unbalanced three-phase conditions, we applied a step change to both the amplitude and phase of the voltage $\mathrm{V}_{\mathrm{a}}$ in Equation (1). This change was defined as a $50 \%$ drop in the amplitude and phase increase of $60^{\circ}$ at $0.4 \mathrm{~s}$.
Figure 5 shows the corresponding evaluation results, where only the DDSRF-PLL successfully tracks the phase of the positive- sequence voltage in approximately $0.05 \mathrm{~s}$. In contrast, the SRF-PLL shows an oscillation in the tracking error despite its lowpass filter, and the FFT-PLL fails to track the phase of the positive-sequence voltage under unbalanced operation, because it is designed to track the phase of the phase-A voltage, where the unbalance was applied.

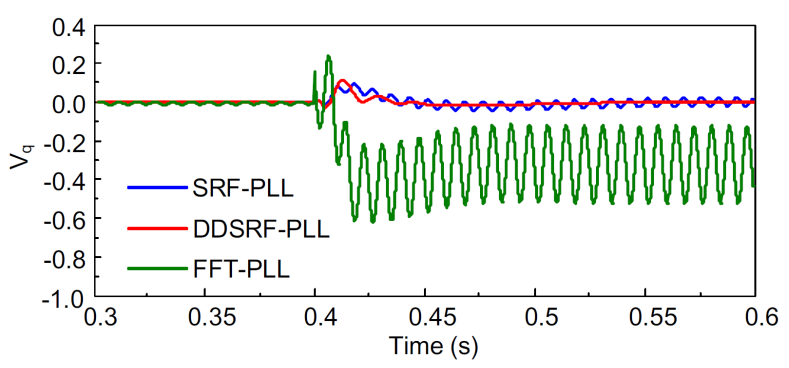

Figure 5. Performance of different PLLs after a step change in amplitude and phase of the phase-A voltage.

\section{Performance Evaluation of PLL Based on Power System Simulation}

To investigate in more detail the PLL performance in a power system, we simulated the power system shown in Figure 6 , which is a typical structure including thermal power gene-ration bundled with a wind farm to supply power to a very large power network through high-voltage transmission lines. The power network is modeled as an infinite bus at point $\mathrm{A}$, thermal generation is modeled as an $\mathrm{SG}$, and the wind farm is repre-sented by a permanent-magnet SG. We applied different faults to assess the performance of the three PLL designs in Section 2.1. The steady-state load is represented by the SG supplying $160 \mathrm{MW}$ of active power and the permanent-magnet SG wind turbine generator providing 10 MW of active power.

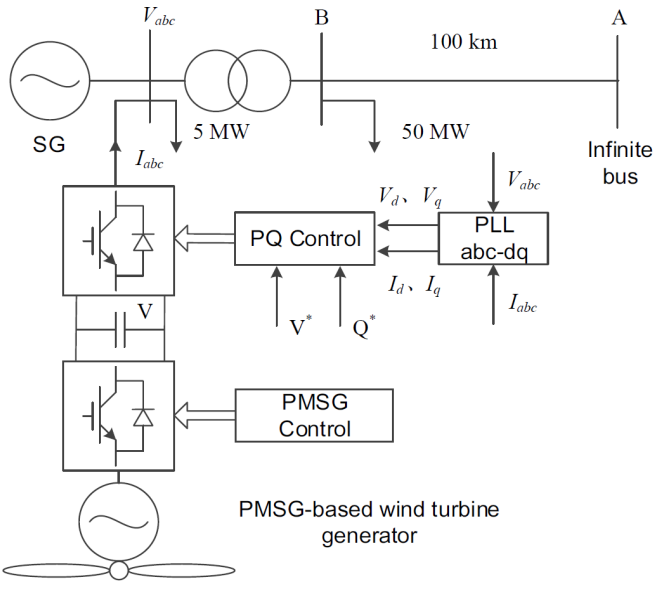

Figure 6. Structure of a power system to evaluate PLL performance (PMSG, permanent-magnet SG). 


\subsection{Load Shedding}

Our first evaluation consisted in shedding the load connected at point B in Figure 6 by $50 \mathrm{MW}$ at $0.3 \mathrm{~s}$ of simulation, thus generating a low-frequency power system oscillation of $1.4 \mathrm{~Hz}$. The simulation results are shown in Figure 7(a), where $V_{q}$ using the SRF- and DDSRF-PLL oscillates around zero at the same frequency of $1.4 \mathrm{~Hz}$, whereas $V_{q}$ using the FFT-PLL is notably polluted by high-frequency noise. To clearly illustrate the behavior of the three PLL designs, Figure 7(b) shows the frequency-domain transformation of the graphs shown in Figure 7(a). The FFT-PLL shows the best performance with the smallest tracking error, whereas the SRFPLL shows the worst performance.

(a) Time domains

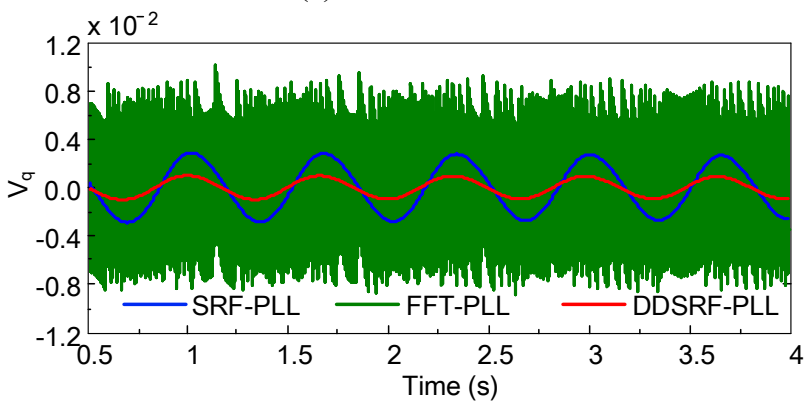

(b) Frequency domains

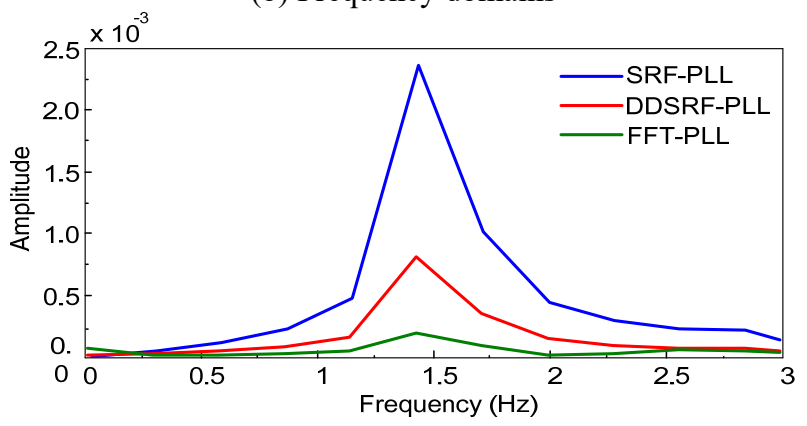

Figure 7. Performance of different PLLs in the power system under load shedding. (a) Time domains; (b) Frequency domains.

\subsection{Single-phase Line Break}

To evaluate the line break effect on the PLL performance, we applied a single-phase line break at the end of a $100-\mathrm{km}$ transmission line at $0.3 \mathrm{~s}$, resulting in a low-frequency power system oscillation of $1.2 \mathrm{~Hz}$. The performance of the SRFand DDSRF-PLL designs under this fault is shown in the time and frequency domains in Figures 8(a) and (b), respectively. The FFT-PLL under this fault does not correctly operate, and hence we did not evaluate it. In the figures, $V_{q}$ varies around zero at $1.2 \mathrm{~Hz}$, and the DDSRF-PLL shows a higher performance than the SRF-PLL. Moreover, $V_{q}$ when using the SRF-PLL exhibits a high-frequency component given the inability of this design to adapt to unbalanced operation, as reported in the previous section.

\subsection{Three-phase Ground Fault}

A three-phase-to-ground short circuit is the worst type of fault as far as the power system first-swing stability is concerned. At $0.3 \mathrm{~s}$ in the simulation, a three-phase-to- ground fault is applied at the point in the transmission line very close to the infinite bus bar, and the fault is cleared in $0.2 \mathrm{~s}$. The simulation results when the performance of the three representative PLL design schemes is assessed are shown in Figure 11.

(a) Time domains

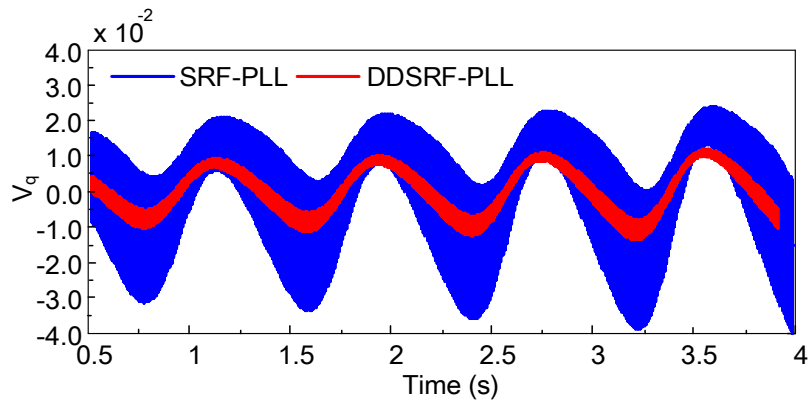

(b) Frequency domains

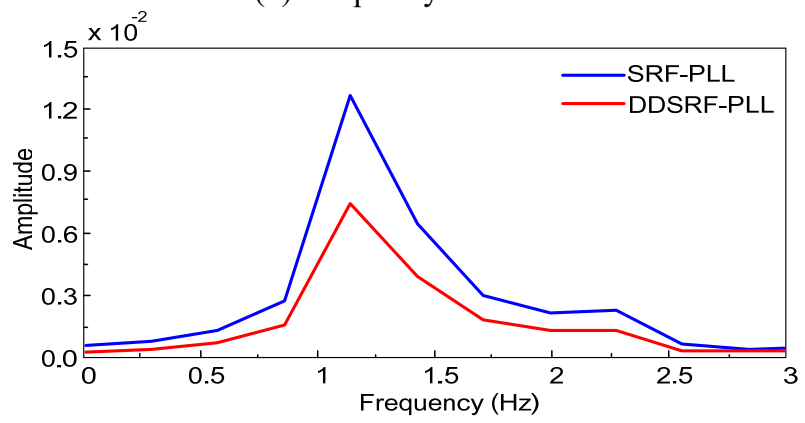

Figure 8. Performance of different PLLs in the power system under single-phase break. (a) Time domains; (b) Frequency domains

During the fault-transient time $(0.3 \sim 0.5 \mathrm{~s})$, Figure $11 \mathrm{sh}$ ows the following: (1) the average value of $V_{q}$ more obviously deviates from zero in both the SRF-PLL and DDSRF-PLL; (2) $V_{q}$ estimated by the SRF-PLL deviates more; and (3) the tracking error of the FFT-PLL is the smallest. Hence, as far as the first-swing stability of the power system is concerned, the performance of the FFT-PLL is the best and that of the SRF-PLL is the worst.

\subsection{Line-to-line Ground Fault}

We then evaluated a line-to-line-to-ground short circuit occurring in $0.3 \sim 0.5 \mathrm{~s}$ when the power system operated under an unbalanced condition, and hence the FFT-PLL was not evaluated. The performance of the other PLL designs is shown in Figure 10(a), where $V_{q}$ using the SRF-PLL is polluted by high-frequency components given its weak adaptability to unbalanced operation. To better visualize $V_{q}$, we applied a mean filter, whose outcome is shown in Figure 10(b). 
Voltage $V_{q}$ using the SRF- and DDSRF-PLL deviates from zero during the fault, but the latter PLL slightly outperforms the former because the average $V_{q}$ is less dev- iated from zero.

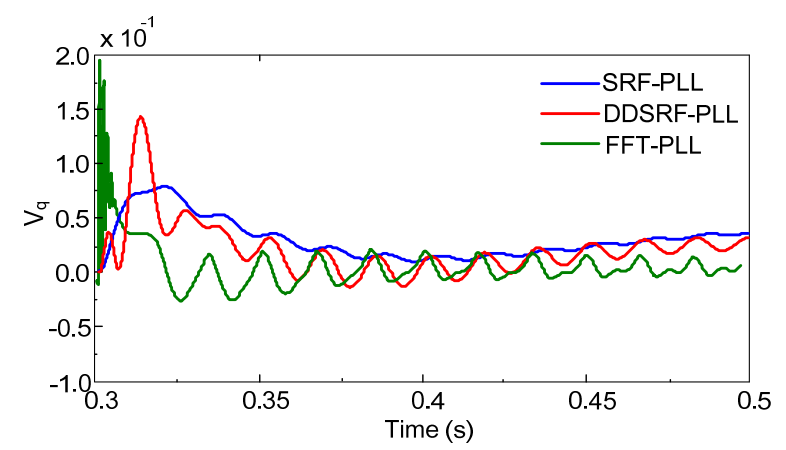

Figure 9. Performance of different PLLs in the power system under three-phase-to-ground short circuit.

(a) Raw results

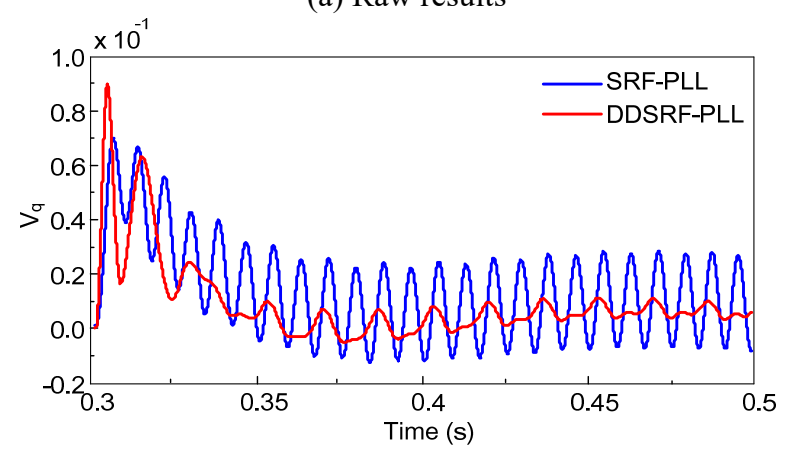

(b) Filtered results

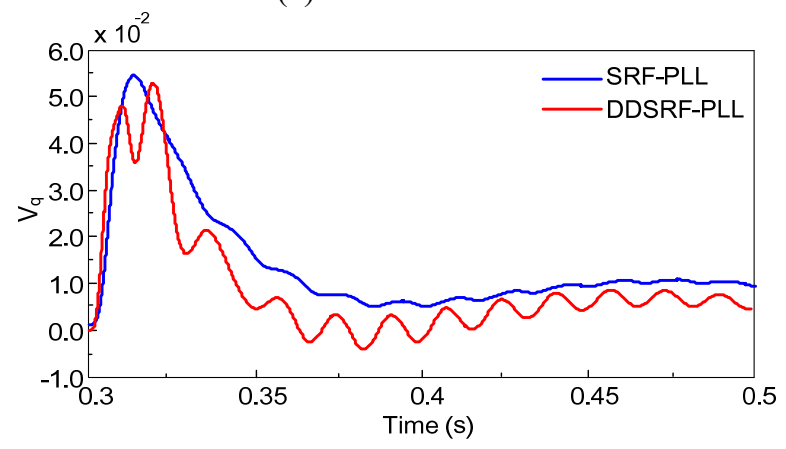

Figure 10. Performance of different PLLs in the power system under line-to-line ground short circuit. (a) Raw results; (b) Filtered results.

\subsection{Discussions}

The results shown in Figures $7 \sim 10$ demonstrate the influence of power system dynamics on the PLL performance. These results cannot be observed under conventional tests considering only connection to a three-phase voltage source, as presented in Section 2.2. In addition, Figure 7 and Figure 8 show that during the dynamic transient of the power system (normally $0.5 \sim 5 \mathrm{~s}$ after a fault), $V_{q}$ oscillates around zero at the low-frequency of the power system. Likewise, Figure 9 and Figure 10 show that during the fault transient $(0 \sim 0.2 \mathrm{~s}$ after a fault), the deviation of $V_{q}$ from zero increases. Overall, these results indicate that the PLL performance requires further investigation. Furthermore, improved PLL designs may be developed by considering the power system dynamics.

The dynamics of a power system reflects electromechanical transients under faults. During the transient, SGs exchange active power with the system by varying the rotor speed to maintain a dynamic balance of active power supply and consumption. The two main concerns during transient are lowfrequency oscillations and first-swing stability. Moreover, the PLL performance under an electromechanical transient may determine the behavior of the VSCs and their effective damping of low-frequency oscillations and preservation of firstswing stability.

For instance, during the dynamic transient of the power system shown in Figure 6 , if the PLL of the permanentmagnet SG fails to track the PCC voltage phase, an extra phase difference will exist between the voltage at the converter terminal and the PCC on the network side. This differrence will result in an additional exchange of active power between the wind generator and the power system, thus affecting the system angular stability. To prevent such scenario, the PLL perfor-mance to track the voltage phase by considering the power system dynamics should be determined. Unfortunately, as demonstrated by the results in Figures 4, 5, and $7 \sim 10$, considering the PLL connected to a three-phase voltage source is not sufficient for a realistic performance evaluation.

On the other hand, evaluating the PLL performance through simulations considering a power system can be timeconsuming. In addition, the PLL performance can vary depending on the configuration, size, and parameters of the simulated power system and the VSCs at different PCCs. In the next section, we propose an efficient method considering different test conditions and the power system dynamics to evaluate the PLL performance. The proposed method includes the system dynamics and different test conditions while considering the PLL connected to a three-phase voltage source for evaluation efficiency.

\section{PLL Performance Evaluation Considering Power System Dynamics}

\subsection{Proposed Evaluation Method}

During electromechanical transient, the power angle within SGs may oscillate, and such low-frequency oscillation in the power system can lead to a weakly or negatively damped oscillation, which can in turn compromise the overall system stability. Let us denote $\Delta \delta$ as the deviation in the power angle of an SG. The dynamic variation of $\Delta \delta$ during low-frequency oscillations of the power system can be expressed as

$$
\Delta \delta=A_{f} \sin \left(2 \pi f_{0} t\right)
$$


where $A_{f}$ is the magnitude and $f_{0}$ is the frequency of the oscillation, which normally ranges between 0.2 and $2 \mathrm{~Hz}$.

In addition, the SG power angle may also increase during a transient and cause system instability during the first swing of the power angle. Let us denote the inertia and magnitude of power unbalance of the SG due to a fault as $M$ and $\Delta P$, respecttively. The rotor motion of the SG is given by (Kundur et al., 1994; Bergen and Vittal, 2000)

$\frac{d^{2} \Delta \delta}{d t^{2}}=\frac{\omega_{0} \Delta P}{M}$

where $\omega_{0}$ is the nominal angular frequency of the power systems. By assuming that $\Delta P$ is almost constant during a fast first-swing transient, we obtain

$\Delta \delta=\frac{\omega_{0} \Delta P}{2 M} t^{2}$

Hence, the deviation in the power angle can be approximately expressed by a quadratic function of time.

Furthermore, the variation in the phase of the PCC voltage mainly follows the change in the SG power angle, especially when the PCC is close to the generator terminal during an electromechanical transient. Therefore, the PLL performance considering the power system dynamics can be determined from Equations (3) and (5) acting on the voltage phase when the PLL is connected to three-phase voltage source, whose expression is given by Equation (1).

\subsection{Evaluation on Balanced Three-phase System}

To verify the effectiveness of the proposed method, we first evaluated the three PLL designs presented in Section 2.1 considering the power system dynamics corresponding to lowfrequency oscillations. To this end, we set the three-phase voltage source in Equation (1) and dynamic variation in equation (3) according to

$\Delta \delta=\left\{\begin{array}{l}0 \quad t<0.4 \text { second } \\ 0.5 \sin [2.8 \pi(t-0.4)] \quad t>0.4 \text { second }\end{array}\right.$

which corresponds to the usually known oscillation parameters. The performance of the PLLs is shown in Figure 11, where $V_{q}$ oscillates around zero at the specified frequency of 1.4 Hz. These results are similar to those in Figures 7(a) and (b) obtained from the power system simulation. Hence, the proposed method suitably reflects the behavior of the PLLs under low-frequency oscillations.

Then, we evaluated the PLL performance considering the power system dynamics on first-swing stability by using Equations (1) and (5) with
$\Delta \delta=\left\{\begin{array}{l}0 \quad t<0.4 \text { second } \\ 29.4524(t-0.4)^{2} \quad t>0.4 \text { second }\end{array}\right.$

which corresponds to the usually known parameters of the SG close to the PCC of the VSCs. The performance of the PLLs is shown in Figure 12, which is similar to that shown in Figure 9 obtained from the power system simulation. Therefore, the continuous increase in the tracking error of the PLL during power system fault transient becomes observable using the proposed method.

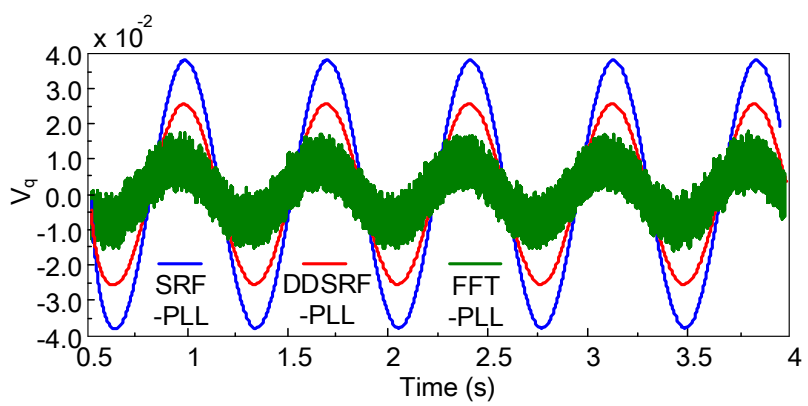

Figure 11. Performance of different PLLs considering power system dynamics under low-frequency oscillation.

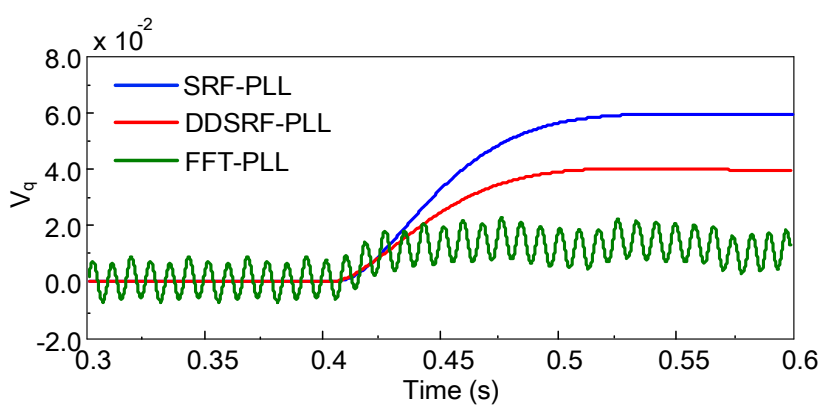

Figure 12. Performance of different PLLs considering power system dynamics and first-swing stability.

\subsection{Evaluation on Unbalanced Three-phase System}

To further verify the effectiveness of the proposed method, we evaluated unbalanced three-phase operating conditions, where the three-phase voltage source in Equation (1) is modified as

$$
\left\{\begin{array}{l}
V_{a}=\left(V_{0}+\Delta V_{0}\right) \cos \left(\omega_{0} t+\Delta \delta+\Delta \varphi\right) \\
V_{b}=V_{0} \cos \left(\omega_{0} t-2 \pi / 3+\Delta \delta\right) \\
V_{c}=V_{0} \cos \left(\omega_{0} t+2 \pi / 3+\Delta \delta\right)
\end{array}\right.
$$

where

$$
\Delta \delta=\left\{\begin{array}{l}
0 \quad t<0.4 \text { second } \\
0.5 \sin [2.8 \pi(t-0.4)] \quad t>0.4 \text { second }
\end{array}\right.
$$


$\Delta V_{0}=\left\{\begin{array}{l}0 \quad t<0.4 \text { second } \\ -0.5 V_{0} \quad t>0.4 \text { second }\end{array}\right.$

$\Delta \varphi=\left\{\begin{array}{l}0 \quad t<0.4 \text { second } \\ -\frac{\pi}{3} \quad t<0.4 \text { second }\end{array}\right.$

to include the power system dynamics associated with the system low-frequency oscillations to resemble unbalanced operation. The performance of the PLLs is shown in Figure 13, where $V_{q}$ using the SRF-PLL is considerably polluted by high-frequency components given its weak adaptability to unbalanced operation. In addition, $V_{q}$ when using both the SRFand DDSRF-PLL oscillates around zero with a frequency of $1.4 \mathrm{~Hz}$. This evaluation is consistent with that shown in Figures 8(a) and (b), whose results were obtained from the power system simulation, thus verifying the ability of the proposed method to reflect the effect of low-frequency oscillations on the PLL performance.

(a) Time domains

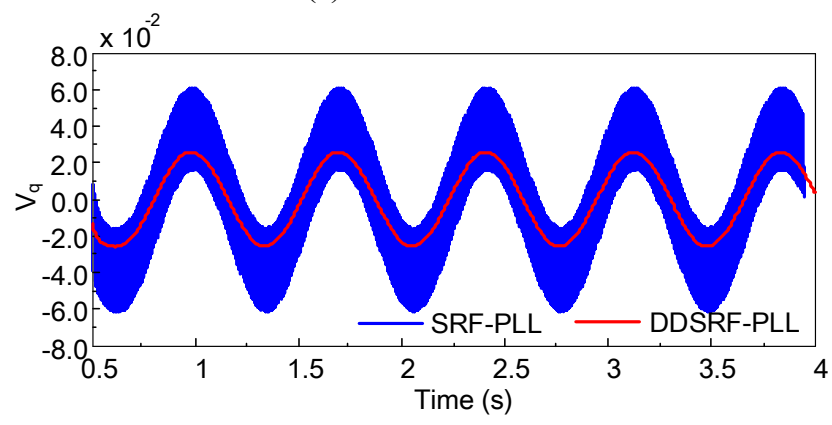

(b) Frequency domains

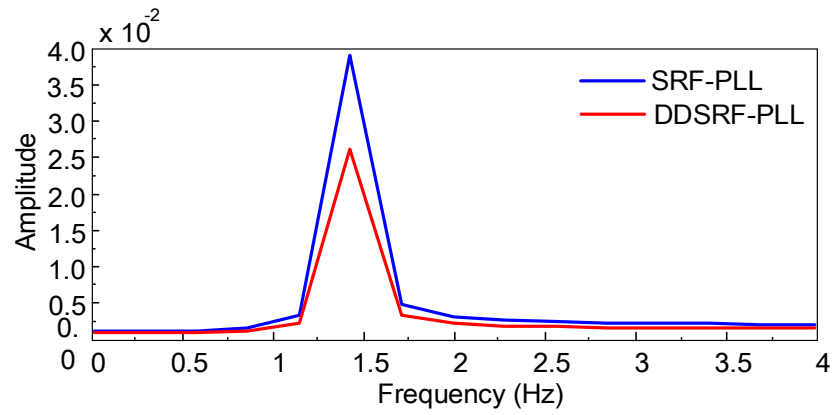

Figure 13. Performance of different PLLs considering power system dynamics under low-frequency oscillation and unbalanced operation. (a) Time domains; (b) Frequency domains.

Finally, to simulate the first swing of the SG under unbalanced operation, we set the three-phase voltage source according to Equations (8) and (10) with

$$
\Delta \delta=\left\{\begin{array}{l}
0 \quad t<0.4 \text { second } \\
29.4524(t-0.4)^{2} \quad t>0.4 \text { second }
\end{array} .\right.
$$

The corresponding performance of the PLLs is shown in Figure 14, which is consistent with Figures 10(a) and (b), thus suggesting that the proposed method suitably models the power system dynamics associated with first-swing stability under unbalanced operation. In fact, the increasing trend in the tracking error of the PLLs is observed when using the proposed method. Overall, the proposed method provides similar results to those obtained from power system simulation but using a more simple and efficient setup.

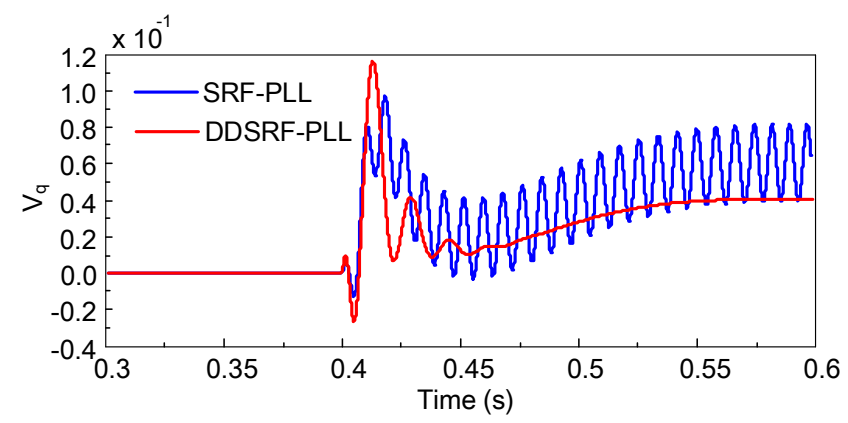

Figure 14. Performance of different PLLs considering power system dynamics and first-swing stability under unbalanced operation.

\section{Conclusions}

PLL design schemes are usually evaluated using simulations or experimental tests with the PLL connected to a three-phase voltage source. Then, test conditions such as step change in the voltage amplitude or phase are applied to verify the PLL performance. However, in a real power system, the dynamic response of the system presents more complex behaviors than these conditions. Hence, the PLL tracking performance under these conditions does not reliably reflect the outcomes from a real power system. To improve PLL evaluation, we considered the power system dynamics instead of the three-phase voltage source. We found that during the dynamic transient of the power system, the tracking error of the PLL varies around zero at the low-frequency power system oscillation. In addition, during the transient of the first swing of the voltage phase, the tracking error of the PLL continuously increases.

These effects on the PLL performance cannot be observed under common test conditions considering the PLL connected to a three-phase voltage source, but they certainly affect the correct implementation of vector control for VSCs and the power system stability. To overcome the limitations of current evaluation methods, we propose an improved method to verify the PLL tracking performance considering power system dynamics. Given that power system simulation is timeconsuming, the proposed method relies on simulations considering the power system dynamics using a single three-phase voltage source. The proposed testing conditions are the following: 
1) Low-frequency oscillation of the voltage phase measured at the PLL to simulate electromechanical oscilla- tions of the power system.

2) Quadratic increase in the voltage phase measured at the PLL to simulate the effects of first swing of the power angle.

In this paper, we verify the limitations of conventional testing conditions to evaluate the PLL performance by connecting it to an infinite busbar and propose the improved evaluation method considering the power system dynamics. The proposed method is applicable to any PLL design scheme and offers high efficiency because its setup is simple com- pared to a power system simulation.

Acknowledgments. This work was supported by the National Basic Research Program of China (973 Program) (2012CB2152 04).

\section{References}

Autom, R. and Co, A.B. (1997). Operation of a phase locked loop system under distorted utility conditions. IEEE Trans. Ind. Appl., 33(1), 58-63. https://doi.org/10.1109/28.567077

Bergen, A.R. and Vittal V. (2000) Power System Analysis, Second Edition. Upper Saddle River, NJ: Prentice-Hall.

Cole, S., Beerten, J., and Belmans, R. (2010). Generalized dynamic VSC MTDC model for power system stability studies. IEEE Trans. Power Syst., 25(3), 1655-1662. https://doi.org/10.1109/TPWRS. 2010.2040846

Da Silva, C.H. and Pereira, R.R. (2010). A Digital PLL scheme for three-phase system using modified synchronous reference frame. IEEE Trans. Ind. Electron., 57(11), 3814-3821. https://doi.org/10. 1109/TIE.2010.2040554

Du, W.J., Bi, J.T., Wang, T., and Wang, H.F. (2015). Impact of grid connection of large-scale wind farms on power system smallsignal angular stability. CSEE J. Power Energy Syst., 1(2), 83-89. https://doi.org/10.17775/CSEEJPES.2015.00023

Du, W., Bi, J., Cao, J., and Wang, H.F. (2016). A method to examine the impact of grid connection of the DFIGs on power system electromechanical oscillation modes. IEEE Trans. Power Syst., 31(5), 3775-3785.https://doi.org/10.1109/TPWRS.2015.2494082

Hadjidemetriou, L. and Kyriakides, E. (2013). A new hybrid PLL for interconnecting renewable energy systems to the grid. IEEE Trans. Ind. Appl., 49(6), 2709-2719. https://doi.org/10.1109/TIA.2013.22 65252

Huang, G.H. and Chang, N.B. (2003). The perspectives of environmental informatics and systems analysis. J. Environ. Inf., 1(1), 1-7. https://doi.org/10.3808/jei.200300001

Kesler, M. and Ozdemir, E. (2011). Synchronous-reference-framebased control method for UPQC under unbalanced and distorted load conditions. IEEE Trans. Ind. Electron., 58(9), 3967-3975. https://doi.org/10.1109/TIE.2010.2100330

Kesler, M. and Ozdemir, E. (2012). Control strategy for three-/four wire- inverter-based distributed generation. IEEE Trans. Ind. Electron., 59(10), 3890-3899. https://doi.org/10.1109/TIE.2012. 2188871

Kundur, P. (1994). Power System Stability and Control. McGrawHill Professional.

Lee, J.P. and Min, B.D. (2011). Active frequency with a positive feedback anti-islanding method based on a robust PLL algorithm for grid-connected PV PCS. J. Power Electron., 11(3), 360-368. https: //doi.org/10.6113/JPE.2011.11.3.360

Lee, K.J. and Lee, J.P. (2014). A novel grid synchronization PLL method based on adaptive low-pass notch filter for grid-connected PCS. IEEE Trans. Ind. Electron., 61(1), 292-301. https://doi.org/ 10.1109/TIE.2013.2245622

Li, S. and Haskew, T. A. (2012). Optimal and direct-current vector control of direct-driven PMSG wind turbines. IEEE Trans. Power Electron., 27(5), 2325-2337. https://doi.org/10.1109/TPEL.2011.2174254

Pena, R. and Clare, J.C. (1996). Doubly fed induction generator using back-to-back PWM converters and its application to variable speed wind-energy generation. IEE Proc. Electr. Power Appl., 143(3), 231-241. https://doi.org/10.1049/ip-epa:19960288

Peng, F. Z. and Lai, J.S. (1997). Dynamic performance and control of a static VAR generator using cascade multilevel inverters. IEEE Trans. Ind. Appli., 33(3), 748-755. https://doi.org/10.1109/28.585865

Rodriguez, P., Pou, J., and Bergas, J. (2005). Double Synchronous Reference Frame PLL for Power Converters Control, 2005 IEEE 36th Power Electronics Specialists Conference, Recife, pp. 14151421. https://doi.org/10.1109/PESC.2005.1581815

Rodríguez, P. and Pou, J. (2007). Decoupled double synchronous reference frame PLL for power converter control. IEEE Trans. Power Electron., 22(2), 584-592. https://doi.org/10.1109/TPEL.2006. 890000

Shi, L. and Crow M. L. (2000). A phase tracking system for three phase utility interface inverters. IEEE Trans. Power Electron., 15(3), 431-438. https://doi.org/10.1109/63.844502

Shi, L. and Crow M.L. (2008). A novel PLL system based on adaptive resonant filter. 2008 40th North American Power Symposium, Calgary, AB, pp. 1-8. https://doi.org/10.1109/NAPS.2008.53 07408

Song, H.S. and Nam, K. (1999). Dual current control scheme for PWM converter under unbalanced input voltage conditions. IEEE Trans. Ind. Electron., 46(5), 953-959. https://doi.org/10.1109/41. 793344

Timbus, A., Teodorescu, R., Blaabjerg, F., and Liserre, M. (2005). Synchronization methods for three phase distributed power genera- tion systems: An overview and evaluation. 2005 IEEE 36th Power Electronics Specialists Conference, Recife, pp. 2474-2481. https:// doi.org/10.1109/PESC.2005.1581980

Wu, D., Lin, C., Perumalla, V., and Jiang, J.N. (2014). Impact of grid structure on dynamics of interconnected generators. IEEE Trans. Power Syst., 29(5), 2329-2337. https://doi.org/10.1109/TPWRS. 2014.2302636

Xu, L. and Andersen, B.R. (2005). VSC transmission operating under unbalanced AC conditions-Analysis and control design. IEEE Trans. Power Deliv., 20(1), 427-434. https://doi.org/10.1109/ TPWRD. 2004.835032 